\title{
Increasing the Societal Impact of Science, Technology, Engineering, and Math with General Collective Intelligence
}

\author{
Andy E. Williams, Nobeah Foundation, Nairobi, Kenya
}

\begin{abstract}
Human-Centric Functional Modeling represents systems in terms of functional state spaces, which enables it to be seen that nature has already solved problems that can be represented in these abstract spaces as being relevant to a wide range of science and engineering disciplines. Namely the same general problem of achieving adaptive general problem-solving ability in any domain can be represented as one that had to be solved by nature's equivalent to engineering and research processes in order for organisms to solve the problem of adapting to any aspect of their environments. This solution can be represented as having been used by nature to address complex problems of adaptation, the equivalent of "wicked problems" in human societies from poverty to climate change. Where these complex problems pose existential challenges, nature has from this perspective not only solved them but has demonstrated the solutions to have worked for hundreds of millions of years. This paper describes how specific patterns in the use of HCFM and General Collective Intelligence (GCI) might be used to approach each of a range of engineering disciplines as well as a range of sciences, in order to significantly increase the societal impact of research and engineering activities.
\end{abstract}

\section{Keywords:}

General Collective Intelligence, STEM, sustainable development goals, systems thinking, systems engineering, education

\section{Introduction}

Within Science, Technology, Engineering, and Math (STEM) as within education in general, technical narrowness, the myth of objectivity, uncritical respect for authority, meritocracy, and the predominance of private sector influence in STEM culture, are said to be primary obstacles to integrating systems approaches into learning that encourage students to think differently about societal problems, because they limit the perspectives students might take [1]. However, fundamental shifts in many core assumptions over the years has created new opportunities to step outside of these limitations. As stated by one researcher, "today's teachers and educational policy makers grew up in a culture characterised by assumptions of unlimited growth, the long-term availability of natural resources(especially of fossil fuels) and a naive assumption that the planet has a carrying capacity that more or less allowed humanity to develop in whatever way it saw fit. These assumptions are now widely challenged" [2]. Students at universities around the world have taken note. A 2018 National Union of Students of the United Kingdom (NUS) survey considering student perceptions of sustainability in higher education clearly shows how undergraduate students are a force that is ready and waiting to be mobilized to make meaningful and tangible contributions now in shaping a sustainable future for themselves and all of us [14].

A General Collective Intelligence or GCI is a hypothetical platform that organizes groups into a virtual collective cognition so they can coherently execute collective reasoning processes in which each participating individual, or each intelligent agent participating on behalf of an individual, executes one or more logical steps in the process [3]. A GCI also enables individuals or such intelligent agents to self-assemble into potentially massive networks of self-sustaining cooperation, in order to create the capacity to exponentially increase the speed and scale of that collective reasoning. In order to achieve this, a GCI leverages Human-Centric Functional Modeling [4] to define a "functional state space" that provides a common representation of concepts and reasoning that might be navigated by the GCI. This 
collective space of concepts or "collective conceptual space" that represents the functional state space of the GCI is hypothesized to be a complete semantic representation of those concepts and reasoning, which in turn is hypothesized to enable understanding rather than just information to be exchanged, so that collective reasoning might occur at vastly greater speed and scale.

While neither a complete GCI nor a complete representation of the collective conceptual space have yet been implemented, a "Social Impact Marketplace" platform approximating a GCI [18], and an ontology within that platform that approximates a small region of the collective conceptual space [24], have already been designed. When applied to conceptual case studies of various social impacts, these case studies support the prediction that the design of this platform has the potential to significantly increase social impact per program dollar.

While the combination of HCFM and GCI are predicted to have fundamentally new capabilities, they are a new combination of largely preexisting concepts. The concept of a "general collective intelligence factor" has been described by many researchers [5], as has the concept that individuals might be organized into collective cognition with vastly greater intelligence than that of any individual [6], [7]. In the academic discipline of logic, a sub-discipline of philosophy, state spaces have long been used to define semantic representations [8]. In engineering, functional modeling is common in large complex projects, where it enables those projects to be more reliably completed by large multidisciplinary teams [9], [10], [11]. Human-Centric Functional Modeling (HCFM) facilitates the use of GCI in the implementation of complex projects by defining a single approach to the functional modeling of systems [4] that is hypothesized to be universal because it depends only on innate human perception.

Patterns in the use of HCFM and GCI described in this paper (table 1) focus on significantly increasing societal impact for specific challenges that might be addressed through STEM [12], such as in civil engineering (sustainable housing) [13], computer science [15], health care informatics [16], and sustainable development [18]. Additional ongoing work continues to explore impacts in a wide range of of other disciplines. As an example, to increase the potential societal impact impact of STEM disciplines on the entire range of sustainable development goals, the design of a ten phase Collective Intelligence based Program to Accelerate Achievement of the Sustainable Development Goals (CIPAASDGs) is being elaborated to leverage GCI to gain the capacity to self-assemble each project into a network of projects that is sustainably self-funding at the scale required to transform communities globally.

Implemented in STEM education, organizing education in units according to these patterns might permit a modular approach that allows for replication with adaptation as experience accrues.

Generalization of learning modules for reuse, and actual reuse of learning modules, creates the opportunity for feedback loops in which one activity has multiple beneficial outputs, and desired outcomes are supported through multiple influencers. These feedback loops might register nonlinear interactions between modules that can not readily be discerned through linear causal analysis. At the project implementation phase following the education phase, these feedback loops might be leveraged to nudge actors toward desired behaviors and away from problematic choices. Through building technical and social mechanisms to regulate flows of information between and within subsystems, it becomes possible to deliver timely, actionable information without overloading the actors. In evaluation of such modular programs, these mappings between systems might be used to understand where critical dependencies occur so its possible to evaluate the programs at those locations. This enables education evaluators to "seek out early indicators of emergent phenomena and conceptualize long-term outcomes in terms of modifications to the larger system of higher education" [17]. 


\section{A GCI Based Pattern for Increasing Societal Impact as Applied to Agriculture}

From the perspective of functional state space, entities such as businesses or projects can be viewed as sets of activities, each of which represents a path through the collective conceptual space. One GCI based pattern through which societal impact might be radically increased is combining businesses into value chains that leverage collectively intelligent cooperation to achieve that increase. This pattern is less relevant to Science, Technology, Engineering, and Math (STEM) because it doesn't involve creation of any significantly new products or services that would involve engineering. One concrete example is the conceptual School Uniform Value Chain in which impact on job creation is significantly increased by launching an entire self-supporting value chain of businesses that can more reliably succeed in creating jobs, and that can reliably create a far larger number of jobs [18].

\section{A GCI Based Pattern for Increasing Societal Impact as Applied to Health Care}

Another GCI based pattern through which societal impact might be even further increased is engineering new GCI based products or services that automate collectively intelligent cooperation, and then leveraging collectively intelligent value chains to increase deployment of those products or services. Automation of collectively intelligent cooperation involves viewing collective activities as paths through the collective conceptual space, where each path segment might be executed by a different entity. Engineering new products and services that automate collectively intelligent cooperation involves identifying these separate roles using a swim lane diagram, and combining activities and roles into new technology that might automate them. This process is said to be based on GCI because this automation functionality is built on top of a GCI platform, or because it at least approximates GCI operating principles that are designed to ensure functionality does not become aligned with the interests of any centralized decision-maker and in doing so becomes unable to adapt in any way necessary to increase impact on collective outcomes. This pattern is relevant to engineering but less relevant to science and math because it doesn't involve research, and therefore doesn't involve any significantly new technologies or theories that might be incorporated into these new products or services that would involve significant scientific or mathematical research. One concrete example is a conceptual Healthcare Provider based Insurance platform that creates the potential to radically decrease healthcare costs through creating an "insurance in a box" platform that automates insurance functions and incorporates those functions into the healthcare provider role [16].

\section{A GCI Based Pattern Applied to Sustainable Housing in Smart Cities}

Yet another GCI based pattern through which societal impact might be yet further increased is leveraging $\mathrm{GCI}$ in the research of new technologies or theories that might be incorporated into the engineering of new GCI based products or services, and then leveraging collectively intelligent value chains to increase deployment of those products or services. Collectively intelligent cooperation in research into better understanding the systems relevant to each discipline involves applying HumanCentric Functional Modeling to represent each domain of behavior of those systems in terms of a separate functional state space, and then defining research problems in terms of gaps through that functional state space. Each new path through functional state space that is discovered then solves the problem of how to transition across the gap from some initial functional state to some target one, where these gaps might be collectively solved through collective reasoning processes in which multiple entities collaborate to each discover a new path segment through the collective conceptual space (to discover new reasoning). This research of new or existing theories is said to be based on GCI because it essentially involves designing research processes that methodically construct research collaboration so that it spreads research activities to explore the entire collective conceptual space regardless of how many or how few researchers are involved. This process is also said to be based on GCI because implementations of processes incorporating any such methodology are built on top of a GCI platform, or because they again at least approximates GCI operating principles that are designed to ensure 
processes do not become aligned with the interests of any centralized decision-maker where doing so prevents those processes from being able to adapt any way required to increase impact on collective outcomes. One concrete example is a conceptual "Design for Change" program in which research into defining semantic models of engineering structures, and research into the development of GCI based design, is used to create design processes capable of organizing vastly larger groups so that they explore a vastly greater design space [18]. This is a form of bio-mimicry of the process in which organisms are able to orchestrate cooperation between massive numbers of cells, whether the 50 trillion or so in a human, or the 100 quadrillion or so in a blue whale, in order to adapt through homeostasis, evolution, or other processes.

\section{Generalization of the GCI Based Patterns for Increasing Societal Impact}

A collectively intelligent value chain can be represented as a path through the collective conceptual space that can be implemented as a collective reasoning process. In a collective reasoning process each participating entity might execute one step in that chain of reasoning. Similarly, in a collectively intelligent value chain each participating business executes a set of activities required of them. To enter into this cooperation each business agrees to purchase a key product or service from another business in the value chain, in return for another business agreeing to purchase a key product or service from it. Businesses at the beginning of the value chain guarantee the demand that supports the entire value chain by utilizing subsidies from the other businesses in the value chain (cross subsidization) to outcompete imports with their local products and services. Businesses form such value chains in order to significantly increase some common social impact. Donors or governments cooperate to support that impact through impact bonds paid through grants in the case of donors, or through tax credits in the case of governments, where these incentives increase returns and/or decrease risk for investors or lenders to whatever degree is required to reliably fund the value chains.

The collective reasoning process corresponding to each specific pattern of value chain might be represented using an ontology, but these value chains can also be represented at a higher level of abstraction in terms of the collective conceptual space. It is hypothesized that a set of only four operations "spans" this entire space in that any reasoning process can be represented as some composition of these four operations. Each of these operations (described elsewhere [19]) act on concepts which serve as the domain object that all processes in the domain consume as input and produce as output. This ability for all processes to be represented in terms of a common set of abstract functions allows this pattern of collectively intelligent cooperation to be extended past chains of businesses to any chains of logic in general, including those that might significantly accelerate and increase the impact of engineering in any engineering discipline, or those that might significantly accelerate and increase the impact of research in any scientific discipline.

Similarly, it is hypothesized that scaling a collectively intelligent value chain can be represented as a path through a "cooperation space" [26], a functional state space with the capacity to represent all possible modes of cooperation through which social outcomes might be scaled. Assuming that the cooperation space can also be spanned by a set of basic operations, where these operations act on activities as their domain object, then cooperation might be defined to scale outcomes to any degree. Value chains might be scaled into programs, and programs might be scaled into sets of programs that enable further value chains to self-assemble within them. The operations hypothesized to span this cooperation space are positively or negatively scaling activities in parallel (parallelization), positively or negatively scaling activities in series (serialization), positively or negatively scaling decentralization (increasing centralization or increasing decentralization), and positively or negatively scaling distributedness. 


\section{Patterns in the Use of Human-Centric Functional Modeling and General Collective Intelligence to Increase the Societal Impact of STEM \\ Name Description \\ "Chains of Cooperation" Increasing Impact on Collective Outcomes through Deployment of \\ (also called "Value \\ Collectively Intelligent Chains of Cooperation (Solve Closed Problem with \\ Chains" in a business \\ context) \\ "Change of Components \\ in Cooperation" (also \\ Increasing Impact on Collective Outcomes through Development of New \\ Collective Intelligence Based Products and Services Deployed in \\ called "Engineering New Collectively Intelligent Chains of Cooperation (Engineer New Products and/ \\ Products or Services" in a or Services as an Open Solution to Solve a Closed Problem) [16] \\ business context) \\ "Change of Targeted Increasing Impact on the SDGs through Collective Intelligence Based \\ Outcome and Components Research of New Technologies Incorporated in the Development of New \\ of Cooperation" (also \\ Collective Intelligence Based Products and Services Deployed in \\ called "Incorporating \\ Research into the \\ Collectively Chains of Cooperation (Research New Technologies and \\ Engineering of New \\ Engineer New Products and/or Services as an Open Solution to Solve an \\ Products or Services" in a \\ business context)}

Table 1: Patterns in the Use of Human-Centric Functional Modeling and General Collective Intelligence to Increase the Societal Impact of STEM

These patterns are a form of bio-mimicry because HCFM assumes that all biological organisms, including humans, can be represented in terms of a hierarchy of systems that each have behavior confined to their own functional state space. The two functional domains included within GCI are the cognitive domain and the cooperation domain represented as also occurring within the human state space.

As mentioned, as a specific example of the "Chains of Cooperation" pattern, the conceptual case study of a school uniform value chain in South Sudan showed the potential to increase collective outcomes (impact on agricultural livelihoods) by seven hundred and fifty times per program dollar [18]. As a specific example of the "Change of Components in Cooperation" pattern, the conceptual case study of a healthcare value chain in the US showed the potential to increase collective outcomes (impact on access to affordable healthcare) through development of GCI based healthcare and health insurance platforms [16]. As a specific example of the "Change of Targeted Outcome and Components of Cooperation" pattern, the conceptual case study of a "Design for Change - Nairobi" sustainable housing design challenged showed the potential for an increase collective in outcomes (impact on access to affordable sustainable housing) by a significant enough margin to impact poverty through research into semantic representation of designs, where that research might be incorporated into a "Collaborative Design Platform" that facilitates collaboration between a massively larger number of designers to discover higher impact outcomes in a far larger region of solution space [13].

The first three phases of a ten phase hypothetical Collective Intelligence based paper to Accelerate Achievement of the Sustainable Development Goals (CIPAA-SDGs) provide examples of how these potential impacts might be extended to a range of outcomes by a series of programs that enables selfsustaining collectively intelligent cooperation to self-assemble between both individual projects and programs consisting of those projects, where this larger cooperation might increase outcomes to the point that impact is sustainably self-funding on the scale required to achieve global transformation. 


\section{CIPAA-SDGs Program}

\section{Phase Description}

I Increasing Impact on the SDGs through Deployment of Collectively Intelligent Value Chains (No New Products or Services)

II Further Increasing Impact on the SDGs through Development of New Collective Intelligence Based Products and Services Deployed in Collectively Intelligent Value Chains (Engineering New Products and/or Services)

III

Still Further Increasing Impact on the SDGs through Collective Intelligence Based Research of New Technologies Incorporated in Af the Development of New Collective Intelligence Based Products and Services Deployed in Collectively Intelligent Value Chains (Research New Technologies)
Targeted Outcome

Increased Agricultural

Livelihoods

Increased Access to Employment as well as to Affordable Education, Healthcare, Renewable Energy, Food Security, Water and Sanitation

Increased Access to

Affordable Sustainable Housing

2: Targeted outcomes of the CIPAA-SDGs paper per phase.

An initiative such as the CIPAA-SDGs program is a potential opportunity to incorporate Vertically Integrated Projects (VIP) into education, where VIP is a new, innovative style of research-based education originally conceived at the Georgia Institute of Technology [14]. Aligning VIP with the SDG framework presents a powerful opportunity for combining both research-based education (RBE) and education for sustainable development (ESD), as a means of effectively embedding research-based education for sustainable development (RBESD) in undergraduate curricula [14].

What is particularly novel about the VIP approach is that research teams consist of undergraduate students ranging from all year groups; encouraging students to work continuously on the same project (gaining academic credit) throughout their University career. This "vertically integrated" approach ensures a constant throughput of students is maintained, where "new generations" of students joining the team can build on the successes of, and learn lessons from, students who have gone before them. Overtime, these VIP teams can reach a critical mass and level of continuity that allows the formation of long-term research groups, consisting primarily of undergraduate students. This allows teams to address such bold and ambitious research challenges [14]. In addition to this vertical approach, programs such as CIPAA-SDGs permit horizontal integration of project teams across disciplines, which opens the opportunity for students to interact with, and teach and learn new skills and gain new knowledge in other fields outside of this. For example, engineering students may be required to explain the basic concepts of power and energy to business students, while business students may be required to explain he basic principles of conducting a market assessment or cost/benefit analysis in return [14].

\section{Directions Forward: Changing STEM Education to Facilitate General Collective Intelligence} In order to test the deployment of this GCI based cooperation, three courses and an undergraduate research experience are currently being designed to provide instruction regarding how GCI might be leveraged to significantly increase the societal impact of STEM, one for each of these patterns. These courses are intended to prepare 100 undergraduate students to participate in a series of Global Innovation Challenges that will use the large size of the research group (100 undergraduates) to crowd source those GCI based solutions by challenging each individual to use this knowledge to design a solution that is projected to most greatly increase impact on the SDG targeted by each challenge. As to be explored in this undergraduate research experience, HCFM and GCI can potentially be used not just 
to create the potential to significantly increase the societal impact of STEM activities, but also to significantly increase the effectiveness of STEM learning, including through the use of GCI to gain the capacity to self-assemble both instructors and learners into massive networks of cooperation that create the potential to significantly increase impact on collective outcomes. Further goals include exploring how to leverage GCI to radically increase capacity to incorporate new information and delivery methods in the engineering curriculum where doing so might significantly improve these STEM societal impact and STEM learning outcomes. Another goal is exploring how to leverage GCI to reduce the effort of collectively vetting this learning guidance and otherwise using GCI to improve learning outcomes, until achieving these improved learning outcomes can eventually be achieved without any specialized GCI training or other specialist expertise.

Through applying Human-Centric Functional Modeling and General Collective Intelligence to education in science and engineering, and through testing the effectiveness of that application, such research must contribute to the general, explanatory knowledge that underlies STEM education in the following areas:

- STEM Learning and Learning Environments: This research must test the use of HCFM as a common methodology for modeling learning in each discipline in order to introduce greater comparability of learning outcomes for the purpose of optimizing STEM learning and in order to optimize use of learning materials to create optimal STEM learning environments.

- Broadening Participation in STEM fields: This research must test techniques for optimizing STEM learning environments as well as optimizing support networks in order to broaden participation in STEM.

- STEM Workforce Development: This research must test how GCI might be used to selfassemble massive networks of self-sustaining cooperation between businesses, non-profits, governments, and other organizations, to develop the STEM workforce by both assessing and driving current science and technology labor trends through these papers and by measuring paper performance.

Part of communicating any understanding is defining a truthful understanding to communicate, another is defining an effective channel to communicate that understanding, yet another is defining the most effective part of that understanding to communicate in each context. To provide an understanding of how Human-Centric Functional Modeling and General Collective Intelligence might be used to significantly increase the impact of research and engineering activities in each of a range of engineering disciplines (mechanical, civil, industrial, computer, and bio-engineering), as well as a range of sciences (initially physics, and biology), and potentially mathematics, courses taught in this project must develop examples specific to each science or discipline, of the patterns through which case studies have suggested this increase in impact might occur. To build each student's capacity to apply this knowledge, research experiences must enable these undergraduates to apply these concepts to the problem of achieving the social, environmental, economic, or other impact most relevant to them and/or their own local communities.

Such a research experience must also explore the patterns of problems that are not believed to be solvable with any current decision-systems other than GCI [21]. This include:

- The "famine problem" (misaligned incentives)

- The "better mousetrap problem" (inability to compare options and reliably select best one)

- The "directions problem" (inability to discern which research direction provides the most productive path forward in the case of wicked problems) 
In exploring the three patterns (defining GCI based value chains, engineering GCI based products or services, GCI based research) for massively increasing capacity to address these problems and massively increasing the societal impact of STEM, such courses and such a research experience must also explore how those patterns might be constructed entirely from proven business management patterns (cross subsidization, economy of scale, value chain redundancy for resilience, service level optimization, etc.) for which there are many case studies in the literature demonstrating their validity.

It is expected that at the early stages such education must emphasize facilitating undergraduate research over teaching because the patterns for increasing societal impact have not been fully implemented and remain to be explored. Some business management pattern or other that turns out to be required for success in implementing these patterns might prove to be missing in any given proof of concept implementation. But since all the business management patterns that might be involved have likely been proven on their own outside of being combined into these patterns, success in implementing these societal impact patterns might be a matter of conducting a sufficient number of trials to explore all possible options to find the combination of those business management patterns that works.

Use of Human-Centric Functional Modeling to define all possible options as occupying a functional space space, and definition of GCI based problem-solving as the process of collectively exploring this space to find a working path is in this sense a model of evolutionary adaptation. Evolution relies on a huge number of individual creatures each trying out different evolutionary pathways until they find one that works. HCFM and GCI also rely on scale. Collectively intelligent cooperation based on GCI involves increasing the number of problems HCFM is being applied to, and using HCFM and GCI to increase the group's ability to reuse solutions across those different problems, until a critical mass of solutions is reached at which massive increase in impact on some collective problem is achieved to demonstrate the effectiveness of the approach. A key part of leveraging HCFM for reuse is model driven engineering [23], [24].

Defining impact in terms of ability to navigate a volume of conceptual space, simple geometric arguments based on the graphs of functional state space suggest that HCFM and GCI create the potential for an exponential increase in societal impact [12], where achieving that that impact as well as solving societal challenges requiring that impact are not otherwise reliably attainable. This is a very strong statement. Instead of making this claim and trying HCFM out on a very small problem to validate it, the goal of such education and research is not to make any claims at all, but to focus on these tremendous possibilities, and to educate about the current understanding while also conducting research to understand better where those possibilities might bear fruit. Trying HCFM and GCI out on a very small sample size and demanding that it produce a solution before it is ready might be the most certain way to ensure it fails. At the same time, given finite funding resources, the goal of such education and research must also be to explore how crowds might be leveraged to radically increase a group's collective ability to explore the largest possible solution space per funding dollar, in order to discover a working solution.

\section{Directions Forward: Accommodating Human Factors in Improving STEM Education}

As a case study, the design of the CIPAA-SDGs program may generate a tremendous number of learning examples. To most effectively communicate how to achieve the targeted impact of the CIPAASDGs program this vast quantity of learning examples might be leveraged with digital learning technology and with the learner profiling techniques available through software platforms to identify the most effective learning examples. This information regarding the learning style of each individual student is an important set of human factors that might be used to improve educational technology. Education and educational research might also apply emerging research regarding the most effective 
styles of delivery as channels to communicate that understanding, as well as regarding how it might be possible to structure learning (especially on-line learning) in a way that makes it possible to define the most effective part of that understanding to communicate to each individual in each learning context.

Some of the categories of delivery that might be explored in such a program are listed below:

$\begin{array}{ll}\text { Category } & \begin{array}{c}\text { Category of Delivery } \\ \text { Category Value }\end{array} \\ \text { Teaching Sequence (in Terms of Level of } & \text { Top Down, Bottom Up } \\ \text { Abstraction) } & \text { Single Perspective and Set of Assumptions, } \\ \text { Openness (Accommodation of Different } & \text { Multiple Perspectives and Sets of Assumptions } \\ \text { Perspectives) } & \text { High, Low } \\ \text { Level of Abstraction } & \end{array}$

Table 3: Category of STEM delivery methods.

In addition to applying categories of delivery methods in order to detect learning patterns in which each delivery method is optimal for each student, other patterns might be applied to arrange material into optimal delivery methods as well. For example, the "Patterns in the Use of Human-Centric Functional Modeling and General Collective Intelligence to Increase the Societal Impact of STEM" (table 1) might be applied to sequence the order in which the STEM learning modules are delivered:

\section{Category}

Fixed Modules

Changeable Modules

\section{Category of Learning Material Description}

Only the sequence and combinations of modules might be changed. The objectives (targeted outcomes) that must be optimized are fixed.

The modules themselves might be changed. The objectives (targeted outcomes) that must be optimized remain fixed.

Changeable Objectives of Modules The modules and the objectives (targeted outcomes) of the modules might also be changed in order to optimize larger outcomes.

Table 4: Category of STEM learning materials.

To define the most effective part of this understanding to communicate in each context, as mentioned, educational programs must also apply General Collective Intelligence to gain the capacity to selfassemble both instructors and learners into networks of cooperation that create the potential to massively increase capacity to target the right message in the right context, in order to increase impact on collective outcomes. One path to doing so involves exploring how an undergraduate research experience paper might implement these self-organizing, impact increasing principles to engage a class of perhaps one hundred while achieving impact on potentially millions through projects like the Global Innovation Challenges.

In addition to leveraging the emerging sciences of Human-Centric Functional Modeling and General Collective Intelligence to identify a single optimal approach towards increasing the social impact of STEM education that is customized to each STEM field and each impact, education must also aim to leverage all available human factors to identify and apply a single approach towards increasing the learning impact of STEM teaching that is customized to each individual, while at the same time massively increasing the number of potential delivery channels, sources of material, and other variables which that optimal approach might be drawn from. Some difficulties with a teaching approach such as one based on GCI that introduces the flexibility to see a great many other viewpoints, is dealing with the expectations on the part of some students of a (traditional) fact-based learning model, with defined 
problems and answers [22]. Predisposition to this specific learning style is only one of many human factors. Considering all such factors methodically might itself require General Collective Intelligence to assess the fitness of each option in achieving its targeted outcome. GCI based education strategies might then be applied to assess the fitness of each category of delivery in the context of the learning characteristics of each student in order to optimize STEM learning. Where this data cannot be collected due to privacy restrictions set by educational policy, using the GCI operating principle of peer-to-peer design that eliminates the possibility of third parties inserting their interests into any interaction, tools might be constructed to enable students to privately use this information to improve their own learning experience without sharing it. Some of the learning characteristics that might be applied are listed below:

\section{Learning Characteristics of Student}

Focus of Attention

[Profile Student According to Maslow's Hierarchy of Needs

(Student's Focus Changes Dynamically)]

Interests

[Pick Interests from Text, Image, Audio, or other List (Student's

Interests Change Dynamically)]

Personality Trait: Openness Open to Multiple Perspectives, Prefer a Single Perspective

Personality Trait: Extroversion Learn Individually, Learn in Groups

Personality Trait: Neuroticism Eliminate Risk of Public Mistakes, Create Opportunity for Public Success

Personality Trait: Conscientiousness High Supervision at Risk of Slowing Learning, Low Supervision at Risk of Non-compliance with Optimum Learning Strategies

Personality Trait: Agreeableness High Interaction to Permit Questioning Instructor, Low Interaction to Prevent Time Wasted from Lack of Questions Career Objectives Optimizing Task Outcome (Operational Role), Optimizing Task Objective (Engineering Role), Optimizing Choice of Objective (Research Role)

Decision Style for Assessing New Type 1 Thinking, Type 2 Thinking Material

Table 5: Category of STEM student learning characteristics.

If Education for Sustainable Development (ESD) is to be integrated into STEM, it will be necessary to engage the teachers involved to understand their motivations for doing so in order to best encourage their participation [2], and it will be necessary to understand and avoid reasons for disengagement. Lack of an explicit research dimension to ESD programs is one factor that has been identified as leading to faculty disengagement [14]. One of the reasons STEM instructors nationwide might choose to engage and to self-assemble in a self-sustaining way into massive networks of cooperation is the opportunity to assemble a massive number of learning examples and to be able to survey all possible categories of delivery methods and assess the fitness of those delivery methods in achieving learning outcomes across a large number and diverse range of students. Such educational programs must test claims regarding the conditions under which this is possible (e.g. management of the learning process is organized in a way that permits measurements of learning outcomes and thus comparison of learning interventions).

\section{Research Limitations}

Using Human-Centric Functional Modeling as a methodology for understanding societal impact or for understanding learning systems is relevant to a number of problems in a wide variety of disciplines [23], [24], [20]. In order to effectively target these problems, General Collective Intelligence is believed to be required to create the capacity for groups to execute collective reasoning processes that 
are reliably able to converge on a single coherent conclusion, and therefore to achieve the significantly increased outcomes of STEM activities that might accompany that conclusion. But a complete implementation of General Collective Intelligence doesn't yet exist. Neither does a complete representation of functional state space. In their absence the subset of GCI that can be built or approximated by an individual or small team must be used to align cooperation between any range of activities or initiatives planned, so that each phase builds the additional GCI infrastructure required for the next phase. The aim of GCI based educational programs is not to put all of the required infrastructure in place for students, but to identify what problems this infrastructure might be used to solve in terms of increasing the social impact of STEM and increasing the learning impact of STEM teaching, and how the efforts to solve these problems must be sequenced with the construction of the GCI itself through follow on projects like the Large Scale international Collaborative REsearch (LSCORE) initiatives. Other challenges to deployment exist. The main challenges to the deployment of VIP programs exist around institutional promotion and acceptance, academic regulations, changes required to existing curriculum, assignment of academic credits, degree accreditation requirements, staff engagement and resource, student recruitment, assessment and supervision [14].

It is important to determine the validity of the concepts described in this paper where they might have the potential to radically increase the impact of STEM education in the ways considered in this paper. However, it is also important to consider that not every channel for research innovation has the capacity to convey all such truths even when validated. One important line of research is to identify patterns of characteristics in organizational structures that create the capacity to assess disruptive innovations in different areas. Some structures and policies by which educational organizations are be constructed might provide channels for research in education that simply do not permit certain innovations to disrupt them, in which case adaptive self-improvement of education might not be reliably achievable. Any organizational model for disseminating the learning from such educational research must then be tailored specifically according to this organizational research as well if any such educational program is to self-improve during its course.

\section{Cooperation Required to Implement GCI in STEM Education}

If the combination of Human-Centric Functional Modeling and General Collective Intelligence have the potential to exponentially increase the general problem-solving ability of groups, and therefore to exponentially increase our ability to solve any problem in general, it is predicted that this can only be accomplished through massive participation. But for massive participation to be possible, GCI and HCFM must be applied to a wide range of disciplines. Part of the goal of any educational program or educational research must then be exploring how to build mind share around the understanding of the possibility of this increase in impact when applied to research, and to attract researchers to participate in a set of Large Scale international Collaborative REsearch (LSCORE) initiatives that have been designed to engage the international research community from across a wide range of disciplines to conduct the research to actually apply HCFM to each basic science and engineering discipline. Part of the goal of any educational program or educational research program must also be to attract participation in the ten phase Collective Intelligence based paper to Accelerate Achievement of the Sustainable Development Goals (CIPAA-SDGs) program expected to build the GCI infrastructure that is required from this HCFM perspective for this exponential increase in STEM's societal impact to be possible. This involves creating mind share around the understanding about how building this infrastructure might be achieved through leveraging the CIPAA-SDGs to gain the capacity to selfassemble sustainable development into a network of projects able to achieve sufficient competitive advantage that deployment is reliably achievable, and that is sustainably self-funding at the scale required to transform communities globally. The LSCORE research outcomes targeted for each phase of the CIPAA-SDGs paper are listed in table 6. 


\begin{tabular}{|c|c|c|}
\hline \multicolumn{3}{|c|}{ LSCORE Outcomes Required to Support CIPAA-SDGs } \\
\hline $\begin{array}{l}\text { CIPA } \\
\text { Phase }\end{array}$ & $\begin{array}{l}\text { s Targeted Outcome of CIPAA-SDGs } \\
\text { paper }\end{array}$ & Required LSCORE Outcomes \\
\hline I & Improve Agricultural Livelihoods & $\begin{array}{l}\text { Build Mind Share About Potential for an } \\
\text { Exponential Increase in Outcomes with } \\
\text { Human-Centric Functional Modeling and } \\
\text { General Collective Intelligence }\end{array}$ \\
\hline II & $\begin{array}{l}\text { Improved Access to Employment, } \\
\text { Affordable Education, Healthcare, } \\
\text { Renewable Energy, Water and } \\
\text { Sanitation, Food Security }\end{array}$ & $\begin{array}{l}\text { Use Human-Centric Functional Modeling to } \\
\text { define Functional State Spaces as Semantic } \\
\text { Representations of Each Domain }\end{array}$ \\
\hline III & Improved Access to Sustainable Hous & $\begin{array}{l}\text { gSemantic Modeling of Physical Objects in } \\
\text { Design }\end{array}$ \\
\hline IV & $\begin{array}{l}\text { Improved Access to Smart } \\
\text { Manufacturing }\end{array}$ & $\begin{array}{l}\text { Define Product Hierarchies as a Functional } \\
\text { Genome }\end{array}$ \\
\hline $\mathrm{V}-\mathrm{X}$ & $\begin{array}{l}\text { Sequence Targeted Outcomes Accordi } \\
\text { to Universal Criteria [e.g. Move throu, } \\
\text { Maslow's Hierarchy of Needs] }\end{array}$ & TBD \\
\hline
\end{tabular}

Such an educational and research experience should be part of an evolving series of events (perhaps occurring on an annual basis) that aim to multiply the impact of this paper funding through seeking additional channels of funding for ongoing iterations from disparate sources that allow the program to be expanded across geographies. Beginning with very simple examples, each subsequent year such and educational or educational research program might continually increase in complexity regarding the material addressed and in the scope regarding the topic it is applied to, so that while the first program might accommodate undergraduate students from first year to those entering their fourth year, the iteration in its second year might also have a separate program to accommodate some students at the masters level, and the iteration in its third year might also have a separate program to accommodate some students at the $\mathrm{PhD}$ level. This $\mathrm{PhD}$ program might integrate with the individual's studies through exploring how this increase in impact might be achieved in their specific doctoral research. In this way, through each year such a program might expand in breadth by extending the experience to more patterns for increasing impact across a greater number of subjects, while also expanding in depth.

One way to continuously expand the depth of these education programs is to gradually transition from the use of ontologies to functional state space based semantic models of all concepts and processes involved, in order to be able to exchange understanding rather than information, and to do so through GCI at vastly greater speed and scale. This means that while a complete semantic implementation is not yet possible because some infrastructure is lacking, a subset of semantic functionality can be implemented and used to implement a greater subset of GCI so that more semantic functionality can be implemented in an iterative way. Another is to define the semantic models of processes to increasingly also be human-centric functional models so that the principles of GCI can be used to increase collective ability to understand and navigate complexity in those systems. This involves establishing cooperation with initiatives such as LSCORE so that learning can continuously be harvested. Some of the cooperation through which such an educational program might continuously increase the depth at which the first course is explored is below: 
Discovering new or existing value chains or networks of value chains to optimize collective outcomes Validating value chains

Implementing value chains

Monitoring and management of value chains

Process improvement for value chains

Storing networks of value chains into a "collective genome"

Applying homeostasis, reproduction, evolution, and other life process algorithms to the network.

Table 7: Steps in expanding the depth of course 1 on HCFM and GCI in value chains.

In the second course that expected cooperation is below:

\section{Cooperation in Expanding the Depth of Course 2 on HCFM and GCI in Product and Service Development}

Developing new products or services or networks of products or services to optimize collective outcomes

Validating products or services

Implementing products or services

Monitoring and management of products or services

Process improvement for products or services

Storing networks of products or services into the "collective genome"

Applying homeostasis, reproduction, evolution, and other life process algorithms to the network.

Table 8: Steps in expanding the depth of course 2 on HCFM and GCI in product and service development.

In the third course that expected cooperation is below:

\section{Cooperation in Expanding the Depth of Course 3 on HCFM and GCI in Research}

Researching new technologies that might be incorporated into new products or services or networks of products or services to optimize collective outcomes

Validating potential of technology for impact

Implementing research of technology

Monitoring and management of research

Process improvement for research

Storing networks of predispositions towards research behaviors into the "collective genome"

Storing networks of research behaviors into the "collective intelligence"

Table 9: Steps in Expanding the Depth of Course 3 on HCFM and GCI in Research.

With the increasing complexity of modern engineering systems comes a corresponding need for engineering graduates with the systems thinking (ST) and systems engineering (SE) skills to design and analyze them. If as suggested, the ST/SE skills of students might be substantially improved through the appropriate interventions, then interventions of the type described in this paper might be threaded throughout select undergraduate courses to substantially improve student's ST/SE skills [25].

\section{Conclusions}

GCI is hypothesized to have the capacity to significantly increase the societal impact of STEM, as well as the impact of STEM education. Taking the lead defined by initiatives such as Vertically Integrated Projects for Sustainable Development (VIP4SD), VIP4SD already provides a number of examples for integrating a GCI based systems strategy for social impact into STEM education. Since VIP4SD has been said to have the potential to operate on a "grander, global scale, with a vision that seeks to 
mobilize, scale-up and coordinate student research and outreach effort on an institutional level, and potentially at national and even international levels through cross-institutional collaboration and partnership" it would seem to be in line with the massive scalability called for by GCI [14].

This article might seem premature since while it's predicted that the efforts of the crowd might selfassemble into a GCI once sufficient mind share has been created, and while GCI is predicted to enable a far greater potential impact on the outcomes of engineering projects, where that increase in impact is not predicted to otherwise be reliably achievable, either the existence of a GCI or of that mind share is a critical part of the equation, and neither has yet been created. For this reason this article has been confined to a theoretical analysis as well as an assessment of steps forward. Whether this article is useful is both a philosophical question and a question of timing. To understand the question of timing, consider the time before the invention of jet engines or jet aircraft. A jet engine might be predicted to exponentially decrease the time of travel as compared to other modes of travel, but how much before its time is coming up with a design of one too early to be useful? Certainly it might not have been useful during the age of sailing ships, but how much infrastructure and mind share would be required before building aircraft utilizing that engine design might be feasible? At what stage of civilization might this infrastructure and mind share be expected to arrive? This is only a question that can be answered for certain in hindsight since roadblocks along an unknown path can't be predicted. Consider that implementing the design of a simple digital watch that costs pennies today might have taken more money than existed in the entire world to implement during medieval times. It also might have taken more time than any regime could have expected to exist. No society that undertook such a long and risky project at the sacrifice of more timely needs like military defense or food security would have been expected to successfully complete such a project regardless of their level of investment. However, the time for GCI might very well be ripe. Taking the car as an example of a ripe opportunity, Henry Ford's design of a factory that could mass produces cars was useful before a network of roads had been built, because the motivation and capability to build those roads was able to come soon after. From this perspective the time for GCI appears to be ripe since all the components for GCI potentially already exist. It's potentially just a matter of recognizing how they fit into this model. Furthermore, because the essentially the same approach that has been used to define a model for GCI has been used to define a functional model of an Artificial General Intelligence (AGI), implementation of any component of either might benefit both. The potential benefits of investing in implementing GCI are then immeasurable if these models are valid. Of course, the absence of unknown roadblocks that delay implementation by months, years, or even decades can't be confirmed until implementation has been successfully completed.

Whether this article is useful is also a philosophical question because of the potential importance of GCI, and because of the potentially limited time window to implement it. GCI is important because without GCI it is predicted that wicked problems like poverty and climate change are currently not reliably solvable due to factors in group organization that prevent the exploration of potentially optimal solutions [21]. The time window of GCI is potentially limited because of an effect called the "technology gravity well" [20] which is predicted to act to increase the centralization of technology more and more, until deployment of a decentralized system of decision-making like GCI is no longer reliably possible, at which point problems requiring decentralized collective optimization in order to maximize outcomes for groups that include entire civilizations, will no longer be reliably achievable. Instead, after having fallen into the technology gravity well it is predicted that societies will be constrained to follow goals aligned with the interests of powerful centralized entities (likely corporations and/or governments) in ways that might openly conflict with the public good, where those entities evolve technology too quickly for any significant number of the public to understand, or for any government regulations or corporate policies to be effective in changing even if they did. 


\section{References}

[1] Tomblin, David, and Nicole Mogul. "STS postures: responsible innovation and research in undergraduate STEM education." Journal of Responsible Innovation 7.sup1 (2020): 117-127. Badurdeen, F., et al. "Systems Thinking for Sustainability: Envisioning Trans-disciplinary Transformations in STEM Education." Learning 11 (2012): 12.

[2] Pitt, James. "Blurring the boundaries-STEM education and education for sustainable development." Design and Technology Education: An International Journal 14.1 (2009).

[3] Andy E. Williams, Defining a Continuum from Individual, to Swarm, to Collective Intelligence, to General Collective Intelligence, In print (2021), Int. J. Of Collaborative Intelligence, in press (2022)

[4] Andy E. Williams, A Revolution in Systems Thinking?, Proceedings 2021 Congress of the World Organization of Systems and Cybernetics (WOSC), in press (2022)

[5] Woolley, Anita Williams, et al. "Evidence for a collective intelligence factor in the performance of human groups." science 330.6004 (2010): 686-688.

[6] Krafft, Peter M. "A simple computational theory of general collective intelligence." Topics in cognitive science 11.2 (2019): 374-392.

[7] Heylighen, Francis, and Marta Lenartowicz. "The Global Brain as a model of the future information society: An introduction to the special issue." (2017): 1-6.

[8] Lloyd, Elisabeth A. "A semantic approach to the structure of population genetics." Philosophy of Science 51.2 (1984): 242-264.

[9] Wan, Jiang, Arquimedes Canedo, and Mohammad Abdullah Al Faruque. "Functional model-based design methodology for automotive cyber-physical systems." IEEE Systems Journal 11.4 (2015): 20282039.

[10] Eisenbart, B., Gericke, K., \& Blessing, L. (2013). An analysis of functional modeling approaches across disciplines. Artificial Intelligence for Engineering Design, Analysis and Manufacturing, 27(3), 281-289. doi:10.1017/S0890060413000280

[11] Kurfman, MA, Stone, RB, Rajan, JR, \& Wood, KL. "Functional Modeling Experimental Studies." Proceedings of the ASME 2001 International Design Engineering Technical Conferences and Computers and Information in Engineering Conference. Volume 4: 13th International Conference on Design Theory and Methodology. Pittsburgh, Pennsylvania, USA. September 9-12, 2001. pp. 267-279. ASME. https://doi.org/10.1115/DETC2001/DTM-21709

[12] Williams, A.E. Are wicked problems a lack of general collective intelligence?. AI \& Soc (2021). https://doi.org/10.1007/s00146-021-01297-8

[13] Williams, A. E. (2020, December 1). General Collective Intelligence as the Emerging Paradigm in Human-Centric Design for Sustainability. https://doi.org/10.31730/osf.io/c4xgs, Accepted to International Sustainable Development Research Society (ISDRS) 2020 
[14] Strachan, Scott Munro, et al. "Using Vertically Integrated Projects to embed research-based education for sustainable development in undergraduate curricula." International Journal of Sustainability in Higher Education (2019).

[15] Andy E. Williams, "Cognitive Computing and its Relationship to Computing Methods and Advanced Computing from a Human-Centric Functional Modeling Perspective", In: Pal R. and Shukla K. P. (eds), SCRS Conference Proceedings on Intelligent Systems, SCRS, New Delhi, India, 2021, pp. 16-33. https://doi.org/10.52458/978-93-91842-08-6-9

[16] Andy E. Williams, General Collective Intelligence as a Paradigm in Healthcare, Accepted to European Healthcare Design (EHD) 2020

[17] Kastens, Kim A., and Cathryn A. Manduca. "Using systems thinking in the design, implementation, and evaluation of complex educational innovations, with examples from the InTeGrate project." Journal of Geoscience education 65.3 (2017): 219-230.

[18] Williams, A. E., Approximating an Artificial General Intelligence or a General Collective Intelligence, International Journal of Collaborative Intelligence, in press (2021)

[19] Williams A.E. (2020) A Model for Artificial General Intelligence. In: Goertzel B., Panov A., Potapov A., Yampolskiy R. (eds) Artificial General Intelligence. AGI 2020. Lecture Notes in Computer Science, vol 12177. Springer, Cham. https://doi.org/10.1007/978-3-030-52152-3 38

[20] Andy E. Williams, General Collective Intelligence as a Platform for Social Technology, Proceedings of the 2nd International Conference on Pervasive Computing and Social Networking [ICPCSN 2022], in print (2022).

[21] Williams, A. E. (2022, February 1). Is Sustainability a Pattern of Solution in a Functional State Space?. https://doi.org/10.31730/osf.io/cb2xh

[22] Hogan, Theodore J., and Paul Kelter. "Discerning the Difference between" Feel Good" and" Real Good": Teaching the Complexity of Sustainable Development." Science Educator 24.1 (2015): 62-69. van der Velden, Maja, Børge Kile Gjelsten, and Siri Moe Jensen. "Sustainability Competence in Computer Science Education." Nordic Journal of STEM Education 5.1 (2021).

[23] Williams, A. E. (n.d.). Towards a Domain Specific Modeling Framework for General Collective Intelligence Platforms. Retrieved from osf.io/preprints/africarxiv/xkvy8

[24] Williams, A. E. (n.d.). Using Models to Capture Patterns for Radically Increasing Social Impact. Retrieved from osf.io/preprints/africarxiv/rqs72

[25] Muci-Kuchler, Karim Heinz, et al. "Incorporating systems thinking and systems engineering concepts in a freshman-level mechanical engineering course." 2020 ASEE Virtual Annual Conference Content Access. 2020.

[26] Williams, A. E. (2022, March 2). General Collective Intelligence as a System for Facilitating Parallel and Distributed Computing. https://doi.org/10.31730/osf.io/m7rfh 